\section{Extinction looms} for many mammals

Nearly one-quarter of the world's carnivores and hoofed mammals have moved closer to extinction since the 1970s.

Moreno Di Marco at Sapienza University of Rome and his colleagues looked at the conservation statuses of about 500 species of carnivores and ungulates over the past 40 years. The researchers found that for every species that saw improvements in status, eight deteriorated. Large animals are also sliding towards extinction faster than their smaller counterparts, and the sharpest declines in conservation status were seen in southeast Asian species.

The authors attribute these shifts to factors such as changes in international trade regulations, hunting, habitat loss and geopolitical events such as the collapse of the Soviet Union, which resulted in the loss of protected areas. Conserv. Biol. http://doi.org/rrs (2014)

\section{ECOLOGY}

\section{Warmer climate} disturbs food web

A study of seabird feathers has revealed how climate change is shifting the food web in the Indian Ocean.

Alexander Bond at the University of Saskatchewan in Saskatoon, Canada, and Jennifer Lavers at the University of Tasmania in Hobart, Australia, inferred the diets of flesh-footed shearwaters (Puffinus carneipes) by looking at ratios of carbon and nitrogen isotopes in the birds' feathers that were collected between 1936 and 2011.

The duo found that levels of heavy isotopes which are present at higher concentrations in species further up the food chain - fell in shearwater feathers over the years, hinting that the birds are eating animals that are lower on the food chain. This could be due to a lack of large fish caused by fishing. Furthermore, the length of the shearwaters' food chain could be shortening because of reduced nutrient flow to the Indian Ocean, owing to a warming climate that is weakening the Leeuwin Current near the western coast of Australia.

Glob. Change Biol. http://doi.org/ $\operatorname{rrp}(2014)$

\section{CANCER \\ High cholesterol in prostate tumours}

Prostate cancer could one day be treated by altering the cancer cells' abnormal cholesterol metabolism.

Ji-Xin Cheng at Purdue University in West Lafayette, Indiana, and his colleagues used Raman spectromicroscopy to analyse lipids inside single cells in tissue samples from people with prostate cancer. The team found that a cholesterol derivative, cholesteryl ester, accumulates inside the most aggressive of the cancer cells, but not in normal prostate cells. This build-up occurs because of the loss of PTEN, a tumour suppressor linked to many cancers.

Treating tumour-bearing mice with small molecules that block the accumulation of cholesteryl ester shrank the tumours and slowed their growth.

Cell Metabol. 19, 393-406 (2014)

\section{OPTICS \\ Hot air guides laser beams}

A channel of hot air could enable high-power laser beams to travel through the atmosphere over long distances - which might be useful for applications such as communications.

High-power beams cannot be precisely focused over many kilometres, because the surrounding air absorbs

COMMUNITY CHOICE

The most viewed papers in science

\title{
NEUROSCIENCE
}

\section{Why warm caresses feel so good}

\section{A HIGHLY READ \\ on www.jneurosci. org in February}

Nerve fibres in human skin that are sensitive to gentle touch are specially tuned to respond to slow, skintemperature strokes.

Rochelle Ackerley at the University of Gothenburg, Sweden, and her colleagues used a robotic probe to stroke the forearms of volunteers at different speeds and temperatures. In one experiment, the researchers recorded the electrical responses of the nerves, called C-tactile fibres, in the skin of 18 participants. In another, they assessed how pleasurable 30 different participants considered each stroke.

The C-tactile fibres fired more frequently, and participants reported more pleasure, when strokes were applied slowly and the probe was close to typical skin temperature. The findings suggest that the fibres have a role in evolutionarily important social interactions that rely on touch, such as in romantic relationships or when nurturing a baby.

J. Neuro. 34, 2879-2883 (2014)

and distorts the radiation. To solve this problem, Howard Milchberg and his colleagues at the University of Maryland in College Park created a conduit in air for the laser. They fired a square-shaped array of four intense, low-power light bursts. The dissipating shots left a channel of hot, dense air, through which the researchers then fired a powerful, focused laser beam. The team showed that the air channel guided the beam through 70 centimetres of air, and calculated that this waveguide could work over longer distances.

Phys. Rev. X 4, 011027 (2014)

Shake to make
power

A device that generates electricity through contact and friction might one day be used to harvest the energy from human motion to charge portable electronics.

Zhong Lin Wang and his colleagues at the Georgia Institute of Technology in Atlanta designed a

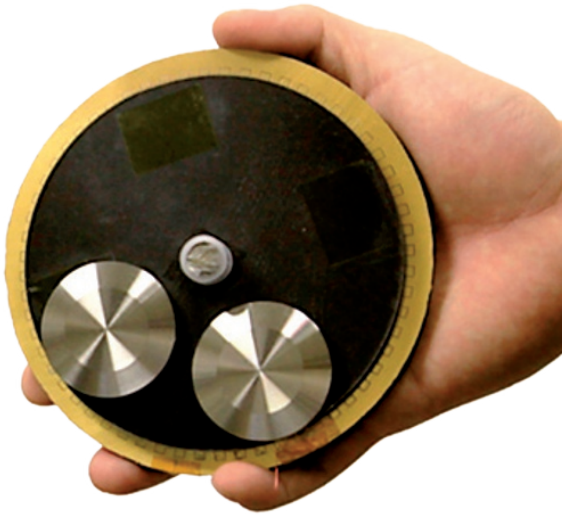

compact, light-weight generator (pictured), consisting of a copperplated disk that spins and rubs against a static base containing a layer of electrodes and a conducting surface. The device can harvest mechanical energy from gentle wind, tap-water flow and normal body movements.

The technology could be developed for large-scale power generation, the authors say.

Nature Commun. 5, 3426 (2014)

\section{DNATURE.COM}

For the latest research published by Naturevisit:

www.nature.com/latestresearch 